\title{
Prenatal diagnosis of congenital high airway obstruction syndrome (CHAOS). Five case report.
}

\author{
Halil Aslan', Ali Ekiz', Deniz Kanber Acar1, Burchan Aydiner², Basak Kaya1, Salim Sezer1
}

${ }^{1}$ Department of Maternal Fetal Medicine, ${ }^{2}$ Department of Obstetrics and Gynecology, Kanuni Sultan Suleyman Education and Research Hospital, Istanbul, Turkey

\section{Abstract}

Congenital high airway obstruction syndrome (CHAOS) is an extremely rare life-threatening condition. Laryngeal atresia appears to be the most frequent cause. Generally the diagnosis is made with severely enlarged and highly echogenic lungs and additional ultrasound findings. The prognosis of the affected infants is often poor. Five cases are reported here that were diagnosed in a tertiary center between 2008 and 2014.

Keywords: congenital high airway obstruction syndrome, laryngeal atresia, ultrasonography

\section{Introduction}

Congenital high airway obstruction syndrome (CHAOS) is an extremely rare life-threatening condition. It occurs as a result of congenital obstruction of the fetal airway secondary to laryngeal and/or tracheal atresia, obstructing laryngeal cysts and obstructing tumors of the oropharynx and the cervical regions. Moreover, laryngeal atresia appears to be the most frequent cause $[1,2]$. The sonographic findings are very characteristic. Generally the diagnosis is made with the 4-chamber view of the fetal heart. Typically, both lungs appear severely enlarged and highly echogenic. The heart points towards the midline of the thorax. Fetal ascites is determined commonly on ultrasound examination.

Five cases of CHAOS, diagnosed antenatally, are being reported here.

Received 16.06.2014 Accepted 06.07.2014

Med Ultrason

2015, Vol. 17, No 1, 115-118

Corresponding author: Ali Ekiz M.D.

Fevzi Cakmak mah. Barbaros cd. 775. Sk

Validesuyu Konutlari C2 blok D:34 Gaziosmanpasa Istanbul/Turkey

Phone: +905059626936

Fax: +90 2125714790

E mail: draekiz@gmail.com

\section{Cases report}

\section{Case 1}

A 25-year-old woman, gravida 2, parity 0 , ectopic pregnancy 1 was referred to our perinatal unit at 19 weeks of gestation on suspicion of a fetal anomaly. There was no history of consanguinity. She and her family had both unremarkable medical histories. Ultrasonography showed enlarged echogenic lungs (fig 1 ), small heart (caused by the compression of the obstructed lungs), dilated trachea, fetal ascites, and flattened diaphragms. There were no other gross abnormalities. The diagnosis of congenital high airway obstruction syndrome (CHAOS) was confirmed.

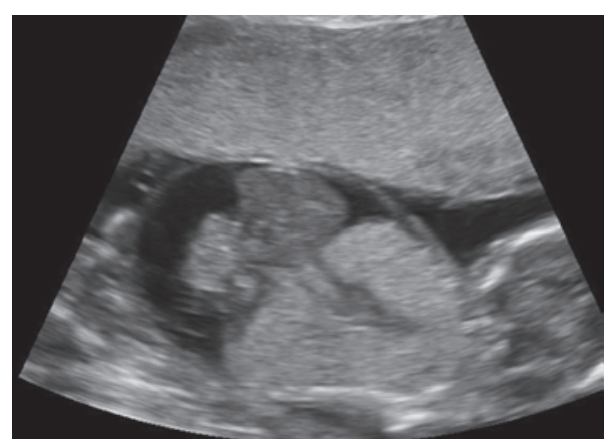

Fig 1. Enlarged echogenic lungs, inverted diaphragm and fetal ascites were detected on ultrasound examination. 
The possible unfavorable outcome of the pregnancy was discussed with the couple. The couple were referred to a genetics specialist and amniocentesis procedure was performed. Fetal demise was determined at 20 weeks of gestation. Fetal karyotype was found normal. The parents refused an autopsy for religious issues.

\section{Case 2}

A 25 -year-old woman, gravida 2 , parity 0 , curettage 1 was referred to our perinatal unit at 20 weeks of gestation for evaluating fetal ascites. There was no history

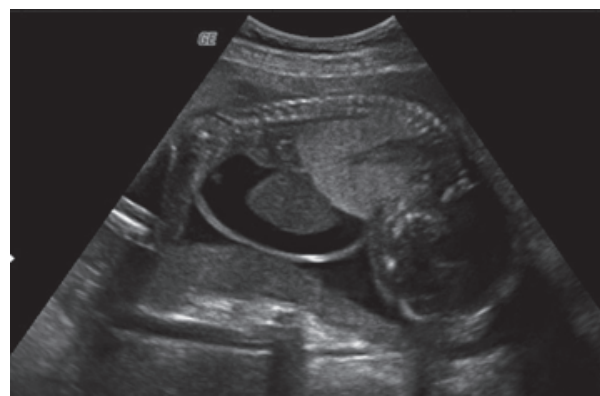

Fig 2. Enlarged echogenic lungs, inverted diaphragm and fetal ascites were shown on sagittal view.

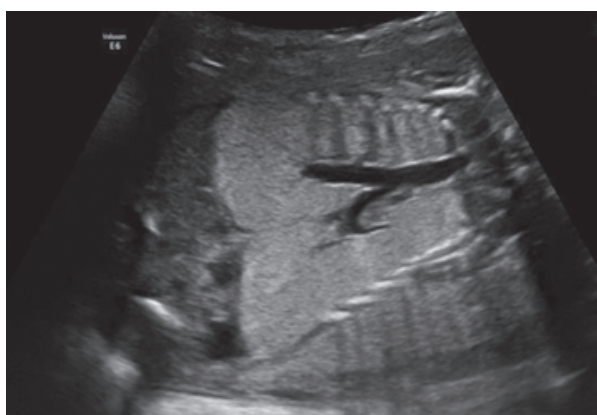

Fig 3. Dilated airways including trachea and main bronchus were demonstrated on coronal view.

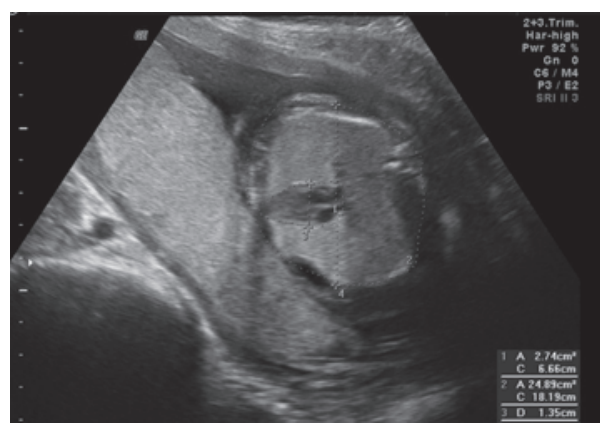

Fig 4. Small and squeezed heart between the lungs and decreased cardiothoracic circumference due to increased intrathoracic pressure were shown. of consanguinity. She and her family had both unremarkable medical histories. Ultrasonographic evaluation showed both lungs had become severely enlarged and highly echogenic. In addition, fetal ascites and inversion of the diaphragm was noted (fig 2). The heart was structurally normal. No other anomalies were noticed.

The possible unfavorable outcome of the pregnancy was discussed with the parents. The couple was referred to a genetics specialist and amniocentesis procedure was offered. The parents refused an amniocentesis and the pregnancy was terminated at the request of the parents. Postmortem examination was not allowed by the family.

\section{Case 3}

A 31-year-old woman, gravida 3, parity 2 was referred at 18 weeks of gestation with severe oligohydramnios. She and her family had both unremarkable medical histories. Both lungs were echogenic, diaphragm flattened, and the heart was compressed. Fetal ascites was also detected. In addition, bilateral renal agenesis and severe oligohydramnios were noted. The main bronchi and trachea were full of fluid (fig 3).

The poor outcome of the pregnancy was discussed with the parents and they underwent genetic counseling. The parents refused fetal karyotyping and chose to receive expectant management. Fetal demise was detected at 28 week gestation and autopsy was declined.

\section{Case 4}

A 29 -year-old woman, gravida 1 , parity 0 woman was referred at 22 weeks with the suspicion of CHAOS. Ultrasonography showed enlarged echogenic lungs, a small heart caused by the compression of the obstructed lungs (fig 4), dilated trachea, fetal ascites and flattened diaphragms. Cardiothoracic $(\mathrm{C} / \mathrm{T})$ circumference was evaluated as 0.36 (normal mean value of 0.45 at 17 weeks and 0.50 at term). There were no other gross abnormalities.

The couple was referred to a genetics specialist and they opted to terminate the pregnancy owing to the severity of the malformation. The pregnancy was terminated at the request of the parents and an autopsy was declined. Fetal karyotype was found to be normal.

\section{Case 5}

A 34-year-old woman, gravida 3, parity 0 , abortion 1 , curettage 1 , was referred to our perinatal unit at 17 weeks of gestation for evaluating fetal ascites. There was no history of consanguinity. She and her family 
had both unremarkable medical histories. Ultrasonographic evaluation showed both lungs had become severely enlarged and highly echogenic. In addition, fetal ascites, inversion of the diaphragm and generalized skin oedema was noted. No other anomalies were noticed.

The possible unfavorable outcome of the pregnancy was discussed with the parents. The couple was referred to a genetics specialist and amniocentesis procedure was offered. But the parents refused an amniocentesis. The pregnancy was terminated at the request of the parents. Postmortem examination was not allowed by the family.

\section{Discussions}

Prenatally diagnosed 12 cases as congenital upper airway obstruction were reported between the years 1988 and 1994. But CHAOS was named by Hedrick et al in 1994 [3]. CHAOS is a rare abnormality, usually with a lethal outcome. CHAOS is responsible for a partial or total obstruction of the upper airways at various levels. It occurs as a result of congenital obstruction of the fetal airway secondary to laryngeal or tracheal atresia, obstructing laryngeal cysts, and obstructing tumors of the oropharynx and the cervical regions. However, laryngeal atresia appears to be the most frequent cause. Differences between laryngeal and tracheal atresia is not feasible on ultrasound examination because both anomalies have the same findings.

CHAOS was defined in association with prenatal ultrasound findings comprising large echogenic lungs, flattened or inverted diaphragms, dilated airways and fetal ascites and/or hydropic fetus. Due to exceedingly high intrathoracic pressure, the heart is squeezed in between the lungs. Identification of a fluid filled trachea and bronchi confirms the diagnosis. However, the findings may not always be typical. Gilboa et al, reported that CHAOS can be diagnosed with transvaginal ultrasound as early as in 15 weeks of gestation, before the development of ascites [4].

Microcystic solid form of congenital cystic adenomatoid malformation (CCAM) and pulmonary sequestration (PS) should be kept in mind as a differential diagnosis. CCAMs are generally unilateral lesions. Unilateral findings of CHAOS highly suggest the diagnosis of bronchial atresia.

There are some genetic syndromes associated with CHAOS such as Short-rib polydactyl syndrome (SRPS), Shprintzen-Goldberg Omphalocele syndrome (SGOS) and VATER/VACTERL association. In addition to these syndromes, some chromosomal abnor- malities (deletions of $22 \mathrm{q} 11.2$, deletion of chromosome 5p [5], 47,XXX, partial trisomy 9 and partial trisomy $16 \mathrm{q}$ ) have been reported in association with CHAOS. Pierre Robin syndrome, the primary developmental defect of the mandible, may be a cause of CHAOS. The other oropharyngeal and neck anomalies are rarely responsible for the CHAOS sequence. CHAOS may occur as part of the Fraser syndrome (tracheal or laryngeal atresia, renal agenesis, microphtalmia, and syn- or polydactyly) [6].

Our five cases were diagnosed in a tertiary center between 2008 and 2014. The diagnoses were made between 17 and 22 weeks of gestations. All fetuses had hydropic findings during the first ultrasound examination. Three fetuses were terminated at the request of the parents. Fetal demise was detected in two cases, one and ten weeks after diagnosis. Bilaterally renal agenesis was detected in one fetus. Amniocenthesis procedure was performed in two cases and normal karyotype was found.

Some intrauterine and intrapartum treatment options are available to manage this condition. It is possible that earlier fetoscopic bronchoscopy may decompress the upper airway and allow an efficient functional lung development [7]. The only available postnatal treatment option to sustain the survival of the fetuses with CHAOS is ex utero intrapartum treatment (EXIT) with tracheal intubation and safe airway control $[8,9]$.

\section{Conclusions.}

Postnatal management of newborn babies with CHAOS is difficult and the prognoses of the affected infants are often poor. The perinatal mortality of CHAOS is $100 \%$ without intervention. Even if there could be a remarkable chance of cure with such treatments as fetoscopic procedures and EXIT, CHAOS is still a high mortality rated anomaly. Even though some genetic syndromes and chromosome aberrations have been reported in association with CHAOS, the actual etiology is unknown. Although successful intrauterine treatments have been reported, the EXIT procedure seems to be the only chance of survival of the affected fetus; therefore, prenatal diagnosis has a vital importance.

\section{References}

1. Sanford E, Saadai P, Lee H, Slavotinek A. Congenital high airway obstruction sequence CHAOS): a new case and a review of phenotypic features. Am J Med Genet A 2012; 158A: 3126-3136. 
2. Roybal JL, Liechty KW, Hedrick HL, et al. Predicting the severity of congenital high airway obstruction syndrome. J Pediatr Surg 2010; 45: 1633-1639.

3. Hedrick MH, Ferro MM, Filly RA, Flake AW, Harrison MR, Adzick NS. Congenital high airway obstruction syndrome (CHAOS): a potential for perinatal intervention. J Pediatr Surg 1994; 29: 271-274.

4. Gilboa Y, Achiron R, Katorza E, Bronshtein M. Early sonographic diagnosis of congenital high-airway obstruction syndrome. Ultrasound Obstet Gynecol 2009; 33: 731-733.

5. Kanamori Y1, Kitano Y, Hashizume K, et al. A case of laryngeal atresia (congenital high airway obstruction syndrome) with chromosome $5 \mathrm{p}$ deletion syndrome rescued by ex utero intrapartum treatment. J Pediatr Surg 2004; 39: E25-E28.

6. Slavotinek AM, Tifft CJ. Fraser syndrome and cryptophthalmos: review of the diagnostic criteria and evidence for phenotypic modules in complex malformation syndromes. J Med Genet 2002; 39: 623-633.

7. Kohl T, Hering R, Bauriedel G, et al. Fetoscopic and ultrasound-guided decompression of the fetal trachea in a human fetus with Fraser syndrome and congenital high airway obstruction syndrome (CHAOS) from laryngeal atresia. Ultrasound Obstet Gynecol 2006; 27: 84-88.

8. Glynn F, Sheahan P, Hughes J, Russell J. Successful ex utero intrapartum treatment (EXIT) procedure for congenital high airway obstruction syndrome (CHAOS) owing to a large oropharyngeal teratoma. Ir Med J 2006; 99 : 242-243.

9. DeCou JM, Jones DC, Jacobs HD, Touloukian RJ. Successful ex utero intrapartum treatment (EXIT) procedure for congenital high airway obstruction syndrome (CHAOS) owing to laryngeal atresia. J Pediatr Surg 1998; 33: 1563-1565. 